\title{
The posttraumatic growth inventory-short form (PTGI-SF): A psychometric study of the spanish population during the COVID-19 pandemic
}

\author{
Helena Garrido-Hernansaiz ${ }^{1}$ Rocío Rodríguez-Rey ${ }^{2} \cdot$ Paula Collazo-Castiñeira $^{2} \cdot$ Silvia Collado $^{3}$ \\ Accepted: 17 December 2021 \\ (c) The Author(s), under exclusive licence to Springer Science+Business Media, LLC, part of Springer Nature 2022
}

\begin{abstract}
People can experience posttraumatic growth (PTG) when faced with potentially traumatic events. One of the most widelyused instruments to measure PTG is the Posttraumatic Growth Inventory-Short Form (PTGI-SF). However, it has not been validated for the Spanish population. This study explored the psychometric properties of the PTGI-SF in adults living in Spain during the COVID-19 pandemic. Since it is a global disaster, two items were added to assess communal PTG. The participants were adult inhabitants of Spain during the COVID-19 pandemic $(N=855)$. They completed the PTGI-SF in July 2020, along with the Impact of Event Scale - Revised to measure symptoms of posttraumatic stress disorder (PTSD). They also rated the degree to which they perceived the COVID-19 crisis as being severe. In November 2020, 592 participants once again completed the PTGI-SF. The factorial validity o was tested by Structural Equations Modeling (SEM). McDonald's $\omega$ coefficients were calculated to test internal consistency. The Intra-class Correlation Coefficient (ICC) was obtained to assess test-retest reliability. Sensitivity and criterion-related validity were assessed by exploring the association of the PTGI-SF scores with gender, age, PTSD symptoms, and perceived severity. Results indicated good psychometric properties for an eight-item, four-factor structure of the inventory in terms of structural validity, reliability, sensitivity and criterion-related validity. These factors were: Relating to Others, Personal Strength, Spiritual Change, and Life Value and Opportunities. Communal PTG overlapped with social PTG, and therefore it was not included. Cultural differences need to be addressed when measuring PTG, especially in terms of spiritual growth.
\end{abstract}

Keywords Posttraumatic Growth • Posttraumatic Growth Inventory · Posttraumatic Growth Inventory-Short Form · PTGI · PTGI-SF; Spain; Spanish

Exposure to potentially traumatic events (PTEs) such as natural disasters, illnesses, and terrorist attacks can have severe psychological consequences. The most frequently reported outcome is posttraumatic stress disorder (PTSD), whose prevalence differs according to several variables such as the nature of the PTE, the degree of exposure, and

Rocío Rodríguez-Rey

rocio.r.rey@comillas.edu

1 Department of Education and Psychology, Centro Universitario Cardenal Cisneros, Alcalá de Henares, Spain

2 School of Human and Social Sciences, Department of Psychology, Universidad Pontificia de Comillas, C/ Universidad Comillas, 3. CP. 28049, Madrid, Spain

3 School of Social and Human Sciences, Department of Psychology and Sociology, Universidad de Zaragoza, Teruel, Spain sociodemographic factors (Neria et al., 2008). However, some people derive benefits and positive changes as a consequence of facing PTEs, such as perceiving themselves to be stronger and giving greater value to their lives, when compared to their value before the PTE (Tedeschi \& Calhoun, 1996). This positive phenomenon is termed posttraumatic growth (PTG) and has been reported by about two-thirds of all individuals who have been exposed to traumatic situations (Helgeson et al., 2006). People report experiencing PTG after exposure to different types of PTE, such as natural disasters (Wlodarczyk et al., 2016), life-threatening illnesses (Sawyer et al., 2010), the critical illness of a child (Rodríguez-Rey \& Alonso-Tapia, 2019), and wars (Lee et al., 2010; Powell et al., 2003). PTG is linked to lower levels of depression in individuals after exposure to a wide variety of PTEs (Helgeson et al., 2006), and to higher satisfaction with life (Mols et al., 2009), optimism, positive 
well-being (Helgeson et al., 2006), and quality of life (Cann et al., 2010a; Liu et al., 2020). It is also linked to the perception that one's life is meaningful (Cann et al., 2010a). Therefore, the evidence demonstrates that PTG is a relevant outcome to be measured in people who have experienced major life crises. The COVID-19 health crisis may be considered a global disaster that, by March $11^{\text {th }}, 2021$ (just one year after the World Health Organization - WHO- declared it as a pandemic), had caused more than 2.6 million deaths worldwide (WHO, 2021). Many studies have explored the psychological impact of this PTE in terms of stress, anxiety, depression, and PTSD. Their collective results show that the COVID-19 pandemic continues to have a massive psychological impact worldwide (e.g., see the meta-analysis by Salari et al., 2020), including Spain (González-Sanguino et al., 2020; Rodríguez-Rey et al., 2020). Fewer studies have explored the prevalence of PTG in connection with the COVID-19 health crisis. Although limited in number, their results indicate that growth may be a positive psychological consequence of the COVID-19 health crisis in different samples, such as the general population in Spain (Prieto-Ursúa \& Jódar, 2020; Vázquez et al., 2021), Greece (Koliouli \& Canellopoulos, 2021) and Turkey (Ikizer et al., 2021), and in specific population groups such as nurses (Chen et al., 2021) and high school students (Yu et al., 2021).

The Posttraumatic Growth Inventory (PTGI) is the most frequently-used tool to measure PTG. It was developed by Tedeschi and Calhoun (1996) and seeks to record PTG in five relevant domains: 1) Relating to Others, which refers to deepened relationships with others, and a greater appreciation of social support after encountering a PTE (e.g., "I have a greater sense of closeness with others"); 2) New Possibilities, referring to the perception of positive changes in the self after experiencing a PTE, which leads to the discovery of new life opportunities (e.g., "I am able to do better things with my life"); 3) Personal Strength, which involves positive changes to self-perception that make the individual feel more capable of overcoming difficulties (e.g., "I discovered that I'm stronger than I thought I was"); 4) Spiritual Change, which refers to strengthened spiritual and/or religious beliefs in the aftermath of a PTE (e.g., "I have a better understanding of spiritual matters"); and 5) Appreciation of Life, or a greater appreciation of one's own existence following the encounter with a PTE and leading to relevant life changes (e.g., "I changed my priorities about what is important in life").

The PTGI consists of 21 items, each rated on a 6-pointLikert scale (ranging from $0=I$ did not experience this change as a result of my crisis to $5=I$ experienced this change to a very great degree as a result of my crisis). In each of the five domains, changes may occur at the affective, cognitive, and behavioral levels. It has displayed adequate internal consistency $(\alpha=0.90)$ and test-retest reliability $(r=0.71)$. The PTGI has been translated into Spanish and validated in this language by Weiss and Berger (2006). The PTGI's five-factor structure has been supported by different studies conducted on multiple populations (e.g., Lee et al., 2010). However, the dimensionality of this construct has been questioned, and the PTGI has been described as being formed by a single factor (e.g., Joseph et al., 2005), three factors (e.g., Powell et al., 2003; Weiss \& Berger, 2006), and four factors (Ho et al., 2004; Taku et al., 2007). These differences may be explained by cultural reasons. While the five original dimensions by Tedeschi and Calhoun (1996) tend to appear in highly individualistic cultures (e. g., Australia), in more collectivist countries (e. g., Guatemala) the dimensions that reflect PTG at a personal level (i.e., New Opportunities, Appreciation of Life and Personal Strength) usually appear as a single dimension (Páez et al., 2013; Wlodarczyk et al., 2017).

Cann and et al., (2010a, 2010b) developed a shortened version of the PTGI: the PTGI-Short Form (PTGI-SF). It measures PTG from 10 items instead of 21, thus significantly reducing the time required by responders to fill in the questionnaire with little loss of information. The PTFISF includes two items for each dimension of the original PTGI. These were selected due to their high loadings on the original factors and the item content. The PTGI-SF displayed adequate internal consistency ( $\alpha=0.86$ to 0.89 ) and confirmatory factor analyses supported the five-factor structure (Cann, et al., 2010a, 2010b). It has been validated in the Spanish language with a sample of Chilean college students, showing a five-factor structure and adequate reliability ( $\alpha=0.83$; Castro et al., 2015). To our knowledge, the psychometric properties of the PTGI-SF have not been studied on adults living in Spain. This is therefore the goal of this study, within the context of the COVID-19 health crisis. We focus on the factorial validity of the scale scores because of the (already described) varying number of factors that are reported in the literature when the PTGI is applied to new populations. Moreover, reliability and evidence of sensitivity and criterion-related validity is also examined.

We also sought to expand research on PTGI by including the growth that may occur at the collective or social level (Páez et al., 2013; Wlodarczyk et al., 2017). Examples of collective PTEs are natural disasters, wars, genocides, and terrorist attacks such as the 9/11 attacks in the United States or $11 \mathrm{M}$ in Madrid (Spain). Given that the COVID-19 pandemic is a global health crisis that affects the entire population, it makes sense to explore the presence of collective PTG. This type of PTG may be especially perceived in cultures with collectivist values, such as Asian and South American cultures (Páez et al., 2013; Wlodarczyk et al., 2017). Spain is a more collectivistic country than the United States, Australia, or the northern and central European nations. There is closer contact with members of the 
community, such as neighbors, than what is experienced in other developed societies, and one's family occupies a central position (Vázquez \& Páez, 2010). Nevertheless, the Spanish culture is considerably more individualistic than Latin American or Asian cultures, to name a few (Vázquez \& Páez, 2010; Weiss \& Berger, 2006). Given Spain's intermediate position within the individualism-collectivism spectrum (Hofstede et al., 2010; Morales et al., 2007), it might be worth exploring whether, in the course of the COVID-19 crisis in Spain, collective growth emerges as a relevant factor different from the Relating to Others dimension. As regards instruments that measure collective PTG, while the PTGI and the PTGI-SF (both originally developed and validated in the United States) do not assess social or collective growth, some other tools to measure PTG do take them into account. For instance, Páez et al. (2012) developed the Community Post-Traumatic Growth (CPTG) scale, which consists of 24 items that explore the presence of PTG at four levels: Personal (e.g., "I have changed my priorities about what is important in life"), Interpersonal (e.g., "I have discovered the support of people who were not close to me"), Social (e.g., "I have discovered that my community, group, or family is stronger than I thought") and Sociopolitical Participation (e.g., "Political and ethical participation and commitments in the country have increased"). By using this scale on the Spanish population during the COVID-19 pandemic, Prieto-Ursúa and Jódar (2020) found similar levels of PTG across its Personal, Interpersonal, and Social/Community dimensions. This suggests that social or collective growth is indeed a consequence of the COVID-19 crisis and hence, this dimension of PTG has also been examined in this study, together with the five dimensions of the PTGI.

Previous studies have shown that PTG is linked to gender, age, perceived severity of the PTE, and PTSD. Two metanalytic reviews concluded that women display higher levels of PTG than men (Helgeson et al., 2006; Vishnesky et al., 2010). Gender-based differences in PTG levels during the COVID-19 pandemic in the Spanish population also support this finding (Prieto-Ursúa \& Jódar, 2020). Results are less consistent when it comes to the link between PTG and age, but most studies show that younger participants tend to report higher PTG levels (Helgeson et al., 2006). Previous studies also found a connection between the perceived severity of the PTE and PTG (e.g., Rodríguez-Rey \& Alonso-Tapia, 2019). It is generally agreed that in order to experience PTG, the event and the emotional response to it must be intense and negative enough to modify the person's basic beliefs. Thus, when a PTE is not perceived as being very severe, disruptive, or traumatic, there is no opportunity for PTG to take place (Helgeson et al., 2006; Janoff-Bulman, 2004). In line with this idea, the current literature on the topic also demonstrates a positive association between PTG and PTSD symptoms, showing that when an event is disruptive enough, both positive and negative psychological consequences tend to co-occur (e.g., metanalysis by Shakespeare-Finch \& Lurie-Beck, 2014). Given these findings, we expect higher PTG scores in women, younger participants, people who perceive the COVID-19 crisis as being more severe, and participants with higher PTSD symptoms. To sum up, the goal of this study is to explore the psychometric properties of the PTGI-SF (extended to include the collective dimension of PTG) when applied to adults living in Spain during the COVID-19 pandemic.

\section{Methods}

\section{Participants}

The participants were over 18 years old and inhabitants of Spain during the COVID-19 pandemic, who could understand the Spanish language well enough to complete the study questionnaires. The inclusion and exclusion criteria were stated in the informed consent form presented before the questionnaire. A total of 855 individuals participated in the study, of whom $77.9 \%$ were women, $21.8 \%$ men, and 3 people stated their gender as Other. The average age was 33.83 years old $(S D=12.57$, range $18-85)$ and $94.5 \%$ of all participants were born in Spain, with the rest usually hailing from other European or Latin American countries. They had a high level of education: $77.2 \%$ held at least a university degree, $12.6 \%$ had received vocational training, 9.6 had completed secondary school, and 4 participants had finished primary school. Most participants were employed (58.2\%), $28.1 \%$ were students, $10.5 \%$ were unemployed, and $3.1 \%$ were retired or had a disability retirement. As regards their marital status, $42.7 \%$ were married or living with a partner, $31.1 \%$ were single, $22.8 \%$ were in a non-cohabiting relationship, $2.9 \%$ were separated or divorced, and 4 participants were widowed. More than a quarter $(28.3 \%)$ had at least one child (among individuals with children, $M=1.79$ children, $S D=0.74$, range $1-5$ ).

\section{Instruments}

Sociodemographic data. The participants reported their gender, age, country of birth, educational level, employment status, marital status, whether they had children and if so, the number of children.

Posttraumatic Growth Inventory - Short Form (PTGI-SF; Cann, et al., 2010a, 2010b). This is a 10-item inventory that measures positive changes after having experienced PTEs in five domains: Relating to Others, New Opportunities, Personal Strength, Spiritual Change, and Appreciation of Life. 
Items are rated on a 6-point Likert-scale $(0=I$ did not experience this change as a result of my crisis; $5=I$ experienced this change to a very great degree as a result of my crisis). This study uses the Spanish translations of the items from the existing 21-item PTGI (Páez et al., 2011) and the wording "the COVID-19 pandemic" instead of "my crisis", to ensure that participants responded according to this PTE. Respondents were given the following instructions: Sometimes, crises like the COVID-19 pandemic entail significant changes in life. Please indicate for each of the statements below the degree to which this change occurred in your life. There are no right or wrong answers [A veces las crisis como la pandemia de COVID-19 suponen cambios importantes en la propia vida. Por favor, indique para cada una de las cuestiones señaladas más abajo si el cambio descrito se ha producido en su vida. No hay respuestas correctas ni incorrectas]. The original short inventory displayed adequate internal consistency ( $\alpha=0.86$ to 0.89; Cann, et al., 2010a, 2010b). As previously indicated, this instrument does not measure Communal Growth, and so two items from the Community Post-Traumatic Growth scale (CPTG; Páez et al., 2012) were included to measure this sixth domain: "I found out that my community, group, or family was stronger than I thought" and "My community, group, or family has become more compassionate or willing to help" (based on the results of Páez et al., 2012, these two items were selected because of their high loading and content).

Impact of Event Scale - Revised (IES-R; Báguena et al., 2001; Gil Moncayo \& Costa Requena, 2007; Weiss, 2007). The IES-R is a 22 -item self-administered tool designed to measure the magnitude of the three main symptoms of PTSD (avoidance, intrusion, and hyperarousal) within the past 7 days, regarding a specific PTE (e.g., "Reminders of it caused me to have physical reactions, such as sweating, trouble breathing, nausea, or a pounding heart"). The instructions were given according to the original format, with COVID-19 specified as the PTE: Below is a list of difficulties people sometimes have after stressful life events. Please read each item, and then indicate how distressing each difficulty has been for you during the past seven days regarding the coronavirus crisis. How much were you distressed or bothered by these difficulties? [Debajo encontrará una lista de síntomas que algunas personas experimentan a veces después de sucesos estresantes. Por favor, lea cada uno e indique cuánto le ha afectado cada uno de ellos en los últimos 7 días con respecto a la crisis del coronavirus. ¿Cuánto malestar ha experimentado por estas dificultades?]. The response format is a 5-point scale ranging from 0 (Not at all or hardly ever) to 4 (A great deal). The IES-R scores have shown adequate psychometric properties in the validation studies (e.g., Báguena et al., 2001), and the internal consistency of the scores in the sample of the current study was excellent $(\alpha=0.94)$.

Perceived severity of the COVID-19 health crisis. Participants indicated the degree of perceived severity of the COVID-19 health crisis on a 10-point Likert scale from $1=$ Not at all severe to $10=$ Very severe .

\section{Procedure}

Data was collected by means of an online questionnaire in July 2020 (about four months after the WHO declared the outbreak a pandemic and the state of emergency was implemented in Spain). Study participants were recruited by email and social networks (Facebook, Instagram, Twitter, LinkedIn, and WhatsApp), following a snowball approach. To explore test-retest reliability, participants were again contacted after four months (November 2020) and asked to complete the PTGI-SF a second time, when we received 592 responses. The study was approved by the ethics committees at the authors' universities and the respondents provided informed consent prior to accessing the questionnaires.

\section{Data Analyses}

To test the factorial validity of the original PTGI-SF plus the two communal growth items (a total of 12 items), two models were specified and analyzed with Structural Equations Modeling (SEM) in order to determine the one that best explained the factorial structure. The one-factor model considered that the 12 items load into a single PTG latent factor. The correlational model considered that there are six first-order factors that are correlated, which correspond to the PTG dimensions: Relating to Others, New Possibilities, Personal Strength, Spiritual Change, Appreciation of Life, and Communal Growth. This second model (minus the Communal Growth dimension) is the one that is supported in the scientific literature (Cann, et al., 2010a, 2010b; Castro et al., 2015). The sample was randomly divided into two $\left(\mathrm{n}_{1}=428 ; \mathrm{n}_{2}=427\right)$ and the models were tested on the first subsample through confirmatory factor analyses (CFA). As the variables were ordinal, we used MLMV as the estimation method (DiStefano, 2002). The fit of the models was assessed through fit indexes (RMSEA, SRMR, CFI, TLI), following standard criteria (SRMR $\leq 0.08$; RMSEA $\leq 0.08$; CFI, TLI $\geq 0.90$; Hair et al., 2016). After the analysis results (e.g., factor loadings, modification indices, and content analysis) were inspected, items were discarded and once again, two models (one-factor and correlated factors) were tested with the retained items. 
The results of the best model were cross-validated through multigroup analyses to test invariance (i.e., to ascertain if it was similarly estimated) across the randomized subsamples $\left(n_{1}=428 ; n_{2}=427\right)$, and across the samples of women $\left(n_{1}=666\right)$ and men $\left(n_{2}=186\right)$. Model fit was again assessed with the RMSEA, SRMR, CFI, TLI indices.

McDonald's $\omega$ coefficients were calculated to test the internal consistency of the dimensions' scores. To assess test-retest reliability, Intra-class Correlation Coefficient (ICC) estimates and their 95\% confident intervals were obtained based on a single-measures, absolute-agreement, 2-way mixed-effects model. ICC is interpreted as follows: poor reliability, $<0.50$; moderate reliability, $0.50-0.75$, good reliability, $>0.75$ (Koo \& Li, 2016).

To study the evidence of sensitivity of the inventory scores, the possible gender and age differences on the PTG dimensions scores and the total score were studied via Student's $t$-tests and Pearson's correlations. Specifically, we ascertained whether these differences resemble those of previous studies (Helgeson et al., 2006; Prieto-Ursúa \& Jódar, 2020; Vishnesky et al., 2010). The evidence of criterionrelated validity was also assessed as follows. As higher posttraumatic stress (Shakespeare-Finch \& Lurie-Beck, 2014) and higher perceived severity of traumatic events (Helgeson et al., 2006; Janoff-Bulman, 2004; Rodríguez-Rey \& AlonsoTapia, 2019) are associated with higher PTG, Pearson's correlations were calculated to examine the association of the PTGI-SF scores with those variables. MPlus 7.2 was used for all SEM analyses and SPSS 25 was used for the rest.

\section{Results}

\section{Confirmatory Factor Analysis}

As displayed in Table 1, the CFA for the one-factor, 12-item PTGI had sub-optimal model fit indices. In turn, the indices for the six correlated factors model were above the standard limits for model acceptance and had the lowest AIC value, indicating a better fit to data. However, a close inspection of the factor loadings, factor correlations, and modification indices suggested that this might not be the best model, since there were extremely high correlations between New Opportunities and Life Appreciation, and between Relating to Others and Communal Growth (in both cases, $r=0.97$, $p<0.001)$. Since the two Communal Growth items did not seem to add to what was already covered by the Relating to Others items, and the content inspection indicated their similarity, we discarded them, thus retaining the original 10 items of the PTGI-SF for further analyses.

We then computed the CFAs for the original 10-item PTGI-SF. Again, the one-factor solution did not fit the data, while the five correlated factors model showed a better fit to data. Once again, close inspection of the factor loadings, factor correlations, and modification indices suggested that New Opportunities and Life Appreciation $(r=0.97$, $p<0.001)$ might function as a single factor. Indeed, the content of those items reflect positive changes in the self, regarding how one perceives life after experiencing a PTE. Moreover, as we discussed, the previous literature supports the unification of dimensions related to personal growth in non-individualistic countries. Thus, we conducted another CFA with those four items in order to examine their factor loadings on that new factor. Items 1,2 and 3 had similar loadings $(\lambda=0.83$ ), while item 6 (establishing a new path in life) had a lower loading $(\lambda=0.67)$. Therefore, we discarded this item. In regard to retaining one item from each original factor, we kept the remaining item from New Opportunities (item 3, ability to do better things with life). Subsequently, we kept item 2 (appreciation of own life's value) and discarded item 1 (changing life priorities) since the content of the former was more in line with item 3 . This new factor covered items 2 and 3 and was labeled Life Value and Opportunities.

New CFAs were then computed with the remaining 8 items. Again, the one-factor solution displayed a bad fit to
Table 1 Goodness of fit statistics of different PTGI models, and multi-group crossvalidation analyses of the best model

\begin{tabular}{|c|c|c|c|c|c|}
\hline & CFI & TLI & RMSEA & SRMR & AIC \\
\hline PTGI 12 items - One factor ${ }^{\mathrm{a}}$ & .79 & .75 & .14 & .08 & $16,891.84$ \\
\hline PTGI 12 items - Six correlated factors ${ }^{\mathrm{a}}$ & .97 & .94 & .07 & .03 & $16,327.35$ \\
\hline PTGI 10 items - One factor ${ }^{\mathrm{a}}$ & .86 & .82 & .13 & .06 & $14,097.37$ \\
\hline PTGI 10 items - Six correlated factors ${ }^{a}$ & .97 & .94 & .08 & .03 & $13,846.33$ \\
\hline PTGI 8 items - One factor ${ }^{\mathrm{a}}$ & .88 & .83 & .14 & .06 & $11,529.29$ \\
\hline PTGI 8 items - Four correlated factors ${ }^{\mathrm{a}}$ & .99 & .99 & .04 & .02 & $11,324.44$ \\
\hline PTGI 8 items - Four correlated factors - MGA-Random ${ }^{\mathrm{b}}$ & .98 & .98 & .05 & .03 & $22,705.99$ \\
\hline PTGI 8 items - Four correlated factors - MGA-Gender ${ }^{c}$ & .98 & .98 & .05 & .04 & $22,525.61$ \\
\hline
\end{tabular}

Note. ${ }^{\mathrm{a}} n_{l}=428 .{ }^{\mathrm{b}}$ Multigroup cross-validation analysis with random subsamples, $n_{1}=428, n_{2}=427 .{ }^{\mathrm{c}}$ Multigroup cross-validation analysis by gender: $n_{l}=666$ (women), $n_{2}=186$ (men). The three participants reporting other gender were excluded from this analysis 
data, while the four correlated factors solution had very good fit indices (see Table 1). Consequently, this model was retained, and its structure (PTGI-8) was used in the rest of the analyses. This model is depicted in Fig. 1, showing the factor loadings, the proportion of item variance explained by the factor, and the correlations among factors.

\section{Sample Invariance}

The PTGI-8 four-factor model was then subjected to two cross-validation analyses, comparing the structure across two randomized subsamples, and across women and men. The fit indices were very similar to those of the model tested in one subsample (see Table 1) and indicated adequate goodness of fit across samples when equality constraints were imposed on factor loadings, factor covariances, observed variable intercepts, and latent means.

\section{Reliability}

As may be seen in Table 2, McDonald's $\omega$ coefficients for the scores of the four PTGI dimensions were all above 0.70, indicating an adequate internal consistency. The same table shows the ICC values for the test-retest reliability, which had a range of $0.56-0.68$, indicating moderate temporal stability of the scores over a four-month period. The PTGI- 8 total score showed good reliability $(\alpha=0.88)$ and moderate test-retest reliability $(\mathrm{ICC}=0.68)$.

\section{Descriptives of the Items}

Table 3 displays the means, standard deviations, asymmetry, and kurtosis of the factor scores of the PTGI-8 items. All the means were around 2 and had similar standard deviations, except for item 8 on religious faith, which had a lower mean. The distribution of items 2 and 3 displayed slightly negative skewness, indicating that data are grouped together slightly above the mean. However, it was the opposite for the rest of the items, especially item 8 (where the most common reply was $0-$ No change). The exception was item 5 ,
Fig. 1 Final standardized solution for the PTGI-8. Correlational model
Table 2 Reliability (omega and ICC), Means, Standard Deviations (SD), Minimum, and Maximum of the Factor Scores of the PTGI-8 Dimensions and PTGI-8 Total

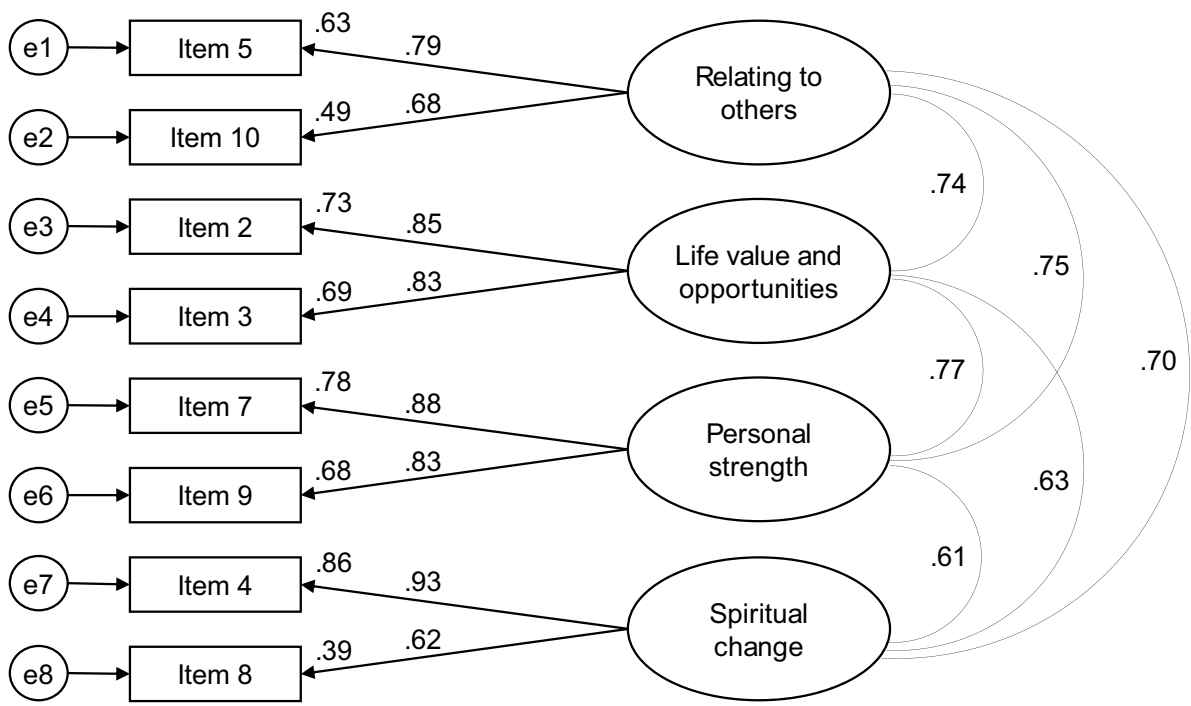

\begin{tabular}{lllllll}
\hline Factor scores & $\omega$ & ICC $(95 \% \mathrm{CI})$ & Mean & $S D$ & Min & Max \\
\hline Relating to others & .70 & $.56(.50-.61)$ & 1.55 & 1.08 & 0 & 3.73 \\
Life value and opportunities & .83 & $.61(.56-.66)$ & 2.16 & 1.27 & 0 & 4.21 \\
Personal strength & .85 & $.59(.53-.64)$ & 1.54 & 1.32 & 0 & 4.27 \\
Spiritual change & .76 & $.68(.63-.72)$ & .96 & 1.06 & 0 & 3.87 \\
PTGI-8 Total & $.88^{1}$ & $.68(.63-.72)$ & 1.55 & .97 & 0 & 3.92 \\
\hline
\end{tabular}

Note. $\mathrm{ICC}=$ Intraclass Correlation Coefficient. $\mathrm{CI}=$ Confidence Interval. 1 For the whole 8-item scale, Cronbach's alpha was computed 
Table 3 Measures of Central Tendency, Dispersion, and Distribution Form for the factor scores on each item of the PTGI-8

\begin{tabular}{|c|c|c|c|}
\hline Items & $M(S D)$ & Skewness & Kurtosis \\
\hline 1. I have a greater appreciation for the value of my own life [Aprecio más el valor de mi propia vida]. (2) & $2.19(1.42)$ & -.23 & -1.17 \\
\hline 2. I am able to do better things with my life [Creo que puedo hacer cosas mejores con mi vida]. (3) & $2.13(1.34)$ & -.29 & -1.12 \\
\hline $\begin{array}{l}\text { 3. I have a better understanding of spiritual matters [Tengo una mejor comprensión de algunas cuestiones } \\
\text { espirituales]. (4) }\end{array}$ & $1.54(1.59)$ & .54 & -1.15 \\
\hline $\begin{array}{l}\text { 4. I have a greater sense of closeness with others [Tengo una mayor sensación de cercanía hacia los demás]. } \\
\text { (5) }\end{array}$ & $1.70(1.28)$ & .04 & -1.28 \\
\hline 5. I know better that I can handle difficulties [Ahora sé mejor que puedo enfrentarme a los problemas]. (7) & $1.55(1.42)$ & .38 & -1.14 \\
\hline 6. I have a stronger religious faith [Tengo una fe religiosa más fuerte]. (8) & $.38(.78)$ & 2.04 & 3.07 \\
\hline $\begin{array}{l}\text { 7. I discovered that I'm stronger than I thought I was [Descubrí que era más fuerte de lo que en realidad } \\
\text { pensaba]. (9) }\end{array}$ & $1.54(1.42)$ & .36 & -1.24 \\
\hline $\begin{array}{l}\text { 8. I learned a great deal about how wonderful people are [Aprendí mucho sobre lo extraordinaria que llega a } \\
\text { ser la gente]. (10) }\end{array}$ & $1.40(1.16)$ & .21 & -1.24 \\
\hline
\end{tabular}

Note. The number in parenthesis at the end of each item wording indicates the original item number in the 10-item PTGI-SF

which displayed almost perfect symmetry. Kurtosis levels revealed that the curves were generally slightly flatter than a normal curve, with the exception of item 8 , which had a peaked curve.

\section{Evidence of Sensitivity and Criterion-related Validity of the PTGI-8 Scores}

Regarding gender differences, Student's $t$-tests showed that women had a significantly higher level of PTG than men in the scores of the four PTG dimensions, as well as in the global PTG score (see Table 4; all $p \leq 0.001$ ). With reference to age, there were significant negative correlations with the PTGI-8 total score and the dimensions Life Value and Opportunities and Personal Strength $(r=-0.13, r=-0.16$, and $r=-0.14$, respectively, all $p<0.001$, see Table 4). Finally, greater PTG (the global score and the four dimensions scores) was significantly associated with higher levels of PTSD symptoms and higher perceived severity of the COVID-19 health crisis.

\section{Discussion}

This study sought to validate the PTGI-SF with a large sample from Spain and to include the dimension of Communal Growth in this measure, in order to explore whether it emerges as a dimension separate from social PTG (i.e., the "Relating to others" dimension). The factorial analyses conducted through SEM showed that no distinction can be made between the PTG domains of New Opportunities and Appreciation of Life in this population. This is consistent with research that shows that these dimensions often emerge as one in collectivist countries (Páez et al., 2013; Wlodarczyk et al., 2017), representing personal growth. However, Personal Strength did not display such a high correlation with them, and was thus retained as a separate dimension. Spain's intermediate position within the individualism-collectivism continuum (Hofstede et al., 2010; Morales et al., 2007) may explain these results that do not conform to either individualistic or collectivistic countries, therefore resulting in a unique configuration.

Table 4 PTGI-8 Associations with Gender, Age, Perceived Severity of the COVID-19 Health Crisis, and PTSD Symptoms

\begin{tabular}{|c|c|c|c|c|c|c|c|}
\hline \multirow[t]{2}{*}{ Factor scores } & \multicolumn{4}{|c|}{ Mean Differences in Gender ${ }^{a}$} & \multicolumn{3}{|c|}{ Correlations } \\
\hline & $\begin{array}{l}\text { Women } \\
M(S D)\end{array}$ & $\begin{array}{l}\text { Men } \\
M(S D)\end{array}$ & $t$ & $p$ & Age & Perceived severity & PTSD symptoms \\
\hline Relating to others & $1.67(1.08)$ & $1.13(.94)$ & 6.61 & $<.001$ & -.05 & $.11 * *$ & $.20 * * *$ \\
\hline Life value and opportunities & $2.27(1.24)$ & $1.77(1.30)$ & 4.71 & $<.001$ & $-.16 * * *$ & $.24 * * *$ & $.36^{* * *}$ \\
\hline Personal strength & $1.67(1.33)$ & $1.09(1.17)$ & 5.75 & $<.001$ & $-.14 * * *$ & $.10^{* *}$ & $.26 * * *$ \\
\hline Spiritual change & $1.02(1.07)$ & $.74(.97)$ & 3.33 & .001 & -.06 & $.11 * *$ & $.23^{* * *}$ \\
\hline PTGI-8 Total & $1.66(.96)$ & $1.18(.93)$ & 5.98 & $<.001$ & $-.13 * * *$ & $.17 * * *$ & $.33 * * *$ \\
\hline
\end{tabular}

Note. ${ }^{\text {a }}$ The four participants reporting other gender were excluded from this analysis

${ }^{*} p<.05$. ** $p<.01 . * * * p<.001$ 
Similarly, no distinction could be drawn between the dimensions of Relating to Others and Communal Growth, suggesting an overlap between interpersonal and communal growth in Spain. Again, this result may indicate that Spain is not collectivistic enough for the Communal Growth dimension to appear separately. This means that the two PTGI-SF Relating to Others items capture interpersonal growth in Spain in a broad sense that appears to include social growth, as both Relating to Others and Communal Growth have some common ground: closeness, social support, etc. Congruently with this idea, Wlodarczyk et al. (2017) included two Relating to Others items that significantly loaded on the Individual Growth factor, but one of them also had a significant and even greater loading on the Communal Growth factor. The authors collected the data in Chile, a country that is much more collectivistic than Spain (Hofstede et al., 2010), and so it seems quite plausible that these two dimensions would function as a single construct in Spain.

A final structure of 8 items was retained (PTGI-8), with four dimensions: Relating to Others, Life Value and Opportunities, Personal Strength, and Spiritual Change. Other similar four-factor structures using the PTGI have been previously reported in the literature on the topic (e.g., Ho et al., 2004; Taku, et al., 2007). This structure displayed a very good fit to data and sample invariance. The four dimensions had good internal consistency and moderate test-retest reliability over a four-month period. Moreover, higher PTG levels were associated with the female gender, younger age, higher perceived severity of the COVID-19 crisis, and higher PTSD symptomatology, results that are consistent with previous studies (Cann, et al., 2010a, 2010b; Helgeson et al., 2006; Janoff-Bulman, 2004; Prieto-Ursúa \& Jódar, 2020; Rodríguez-Rey \& Alonso-Tapia, 2019; Shakespeare-Finch \& Lurie-Beck, 2014; Vishnesky et al., 2010). This finding supports the ability of the PTGI-8 inventory to discriminate between populations with different PTG levels and provides evidence of sensitivity and criterion-related validity.

It is worth paying further attention to the dimension of Spiritual Change, given that its mean score was lower than the scores of the other dimensions. This finding is in line with studies conducted on specific population groups in Spain, such as the parents of critically ill children (Rodríguez-Rey \& Alonso-Tapia, 2019) and earthquake survivors (Wlodarczyk et al., 2016). It is consistent with research showing that Spiritual Change is less pronounced in Western societies than in more religious cultures such as Latin American and African cultures (Páez et al., 2013; Wlodarczyk et al., 2016). This cultural variability has been acknowledged in recent research and is the cause of some concern, since there are only two Spiritual Change items in both the PTGI and the PTGI-SF, and one of them refers explicitly to religious faith (item 18 in the PTGI and 8 in the PTGI-SF). This may pose a problem in cultures that do not appear to be very religious (Tedeschi et al., 2017; Vázquez \& Páez, 2010). This is the case of Spain, where only $27 \%$ of the population consider themselves to be very religious (Vázquez \& Páez, 2010). Indeed, item 8 displayed a very low mean and high values for skewness and kurtosis in our sample. As a recent study on PTG following the COVID-19 health crisis in Spain has shown, both spirituality and religiosity continue to be relevant in the Spanish context (Prieto-Ursúa \& Jódar, 2020). However, without denying this importance, future research conducted in Western societies or with nonreligious individuals may consider other options for this item that are better able to capture the broader and more existential nature of spiritual growth experienced by non-religious individuals. This wider sense of spirituality has already been considered in newer versions of the original PTGI (Tedeschi et al., 2017) and its inclusion in the PTGI-SF might help shed some light on the possible differences in PTG as experienced by religious and non-religious people.

As usual, the strengths and limitations of this study must be noted. Among its strengths, we may mention the relatively big sample of the Spanish adult population, with a wide diversity of ages represented, which permitted multigroup analyses to test sample invariance. However, women were overrepresented in our sample (something quite common in research, Dunn et al., 2004), as well as people born in Spain. Thus, further research with a more gender-balanced sample is needed and until then, the findings should not be generalized to other genders or to immigrants. Moreover, given the online sampling and data collection method, there was little opportunity for people who do not use social media or electronic devices to be recruited into the study. Additionally, all our participants were asked to focus on the COVID19 health crisis, which needs to be considered before our results may be generalized to different PTEs. Finally, more evidence of validity (especially regarding convergent and predictive validity) of the scores of this Spanish PTGI-SF must be gathered.

In conclusion, our study is the first to explore the psychometric properties of the PTGI-SF in Spain, and we found solid evidence of structural validity for an 8-item version of the inventory within the context of the COVID-19 pandemic. The instrument also demonstrated good reliability, sensitivity, and criterion-related validity. Our research provides more data on possible cultural differences in PTG between countries that fall within the individualistic-collectivistic continuum, especially with regard to spiritual growth.

Acknowledgements The authors would like to thank the survey participants for their time and effort in filling the questionnaire during these difficult times.

Authors' contributions All authors contributed to the study conception design, material preparation and data collection. Data analyses were performed by Helena Garrido-Hernansaiz and reviewed by all the 
authors. The first draft of the manuscript was written by Helena Garrido Hernansaiz and Rocío Rodríguez Rey and all authors commented on previous versions of the manuscript. All authors read and approved the final manuscript.

Funding This research was funded by Universidad Pontificia Comillas, grant number PP2020_04.

Availability of data and material The dataset generated during the current study is available from the corresponding author when requested on reasonable request.

Code availability M-plus 7.2 and SPSS 25 were used to perform the data analyses.

\section{Declarations}

Conflicts of Interests The authors declare no conflict of interests.

Ethics approval The study was approved by the ethics committees at Universidad Pontificia Comillas and Centro Universitario Cardenal Cisneros and was performed according to the ethical standards laid down in the 1964 Declaration of Helsinki and its later amendments.

Consent to participate All individuals completed an informed consent prior to participate in this study.

Consent for publication Not applicable.

\section{References}

Báguena, M., Villarroya, E., Belena, A., Amelia, D., Roldán, C., \& Reig, R. (2001). Propiedades psicométricas de la versión Española de la Escala Revisada de Impacto del Estresor (EIE-R) [Psychometric properties of the Spanish version of the Impact of Event Scale-Revised (IES-R)]. Análisis y Modificación de Conducta, 27(114), 581-604. http://hdl.handle.net/11162/25035

Cann, A., Calhoun, L. G., Tedeschi, R. G., \& Solomon, D. T. (2010a). Posttraumatic Growth and Depreciation as Independent Experiences and Predictors of Well-Being. Journal of Loss and Trauma, 15(3), 151-166. https://doi.org/10.1080/15325020903375826

Cann, A., Calhoun, L. G., Tedeschi, R. G., Taku, K., Vishnevsky, T., Triplett, K. N., \& Danhauer, S. C. (2010b). A short form of the Posttraumatic Growth Inventory. Anxiety, Stress \& Coping, 23(2), 127-137. https://doi.org/10.1080/10615800903094273

Castro, M. C., Delgado, J. B., Alvarado, E. R., \& Rovira, D. P. (2015). Spanish Adaptation and Validation of the Posttraumatic Growth Inventory-Short Form. Violence and Victims, 30(5), 756-769. https://doi.org/10.1891/0886-6708.vv-d-13-00165

Chen, R., Sun, C., Chen, J. J., Jen, H. J., Kang, X. L., Kao, C. C., \& Chou, K. R. (2021). A Large-Scale Survey on Trauma, Burnout, and Posttraumatic Growth among Nurses during the COVID-19 Pandemic. International Journal of Mental Health Nursing, 30(1), 102-116. https://doi.org/10.1111/inm.12796

DiStefano, C. (2002). The Impact of Categorization with Confirmatory Factor Analysis. Structural Equation Modeling: A Multidisciplinary Journal, 9(3), 327-346. https://doi.org/10.1207/S15328007S EM0903_2

Dunn, K. M., Jordan, K., Lacey, R. J., Shapley, M., \& Jinks, C. (2004). Patterns in Consent in Epidemiological Research: Evidence from Over 25,000 Responders. American Journal of Epidemiology, 159(11), 1087-1094. https://doi.org/10.1093/aje/kwh141
Gil Moncayo, F. L., \& Costa Requena, G. (2007). Propiedades psicométricas de la escala revisada del impacto del evento estresante (IES-R) en una muestra española de pacientes con cáncer. Análisis y Modificación de Conducta, 33(149), 311-331. http://hdl.handle. net/10272/5848

González-Sanguino, C., Ausín, B., Castellanos, M. Á., Saiz, J., LópezGómez, A., Ugidos, C., \& Muñoz, M. (2020). Mental health consequences during the initial stage of the 2020 Coronavirus pandemic (COVID-19) in Spain. Brain, Behavior, and Immunity, 87, 172-176. https://doi.org/10.1016/j.bbi.2020.05.040

Hair, J. F., Hult, G. T. M., Ringle, C., \& Sarstedt, M. (2016). A primer on partial least squares structural equations modeling (PLSSEM). Sage.

Helgeson, V. S., Reynolds, K. A., \& Tomich, P. L. (2006). A metaanalytic review of benefit finding and growth. Journal of Consulting and Clinical Psychology, 74(5), 797-816. https://doi.org/10. 1037/0022-006X.74.5.797

Ho, S. M. Y., Chan, C. L. W., \& Ho, R. T. H. (2004). Posttraumatic growth in Chinese cancer survivors. Psycho-Oncology, 13(6), 377-389. https://doi.org/10.1002/pon.758

Hofstede, G., Hofstede, G. J., \& Minkov, M. (2010). Cultures and organizations: software of the mind: intercultural cooperation and its importance for survival ( $3^{\text {rd }}$ ed.). McGraw Hill.

Ikizer, G., Karanci, A. N., Gul, E., \& Dilekler, I. (2021). Post-traumatic stress, growth, and depreciation during the COVID-19 pandemic: Evidence from Turkey. European Journal of Psychotraumatology, 12(1), 1872966. https://doi.org/10.1080/20008198.2021.1872966

Janoff-Bulman, R. (2004). Posttraumatic growth: Three explanatory models. Psychological inquiry, 15(1), 30-34. https://www.jstor. org/stable/20447198

Joseph, S., Linley, P. A., \& Harris, G. J. (2005). Understanding positive change following trauma and adversity: Structural clarification. Journal of Loss and Trauma, 10(1), 83-96. https://doi.org/10. 1080/15325020490890741

Koliouli, F., \& Canellopoulos, L. (2021). Dispositional Optimism, Stress, Post-traumatic stress Disorder and Post-traumatic Growth in Greek general population facing the COVID-19 crisis. European Journal of Trauma \& Dissociation, 5(2), 100209. https://doi. org/10.1016/j.ejtd.2021.100209

Koo, T. K., \& Li, M. Y. (2016). A Guideline of Selecting and Reporting Intraclass Correlation Coefficients for Reliability Research. Journal of Chiropractic Medicine, 15(2), 155-163. https://doi. org/10.1016/j.jcm.2016.02.012

Lee, J. A., Luxton, D. D., Reger, G. M., \& Gahm, G. A. (2010). Confirmatory factor analysis of the posttraumatic growth inventory with a sample of soldiers previously deployed in support of the Iraq and Afghanistan wars. Journal of Clinical Psychology, 66(7), 813-819. https://doi.org/10.1002/jclp.20692

Liu, Z., Doege, D., Thong, M. S. Y., \& y Arndt, V. (2020). The relationship between posttraumatic growth and health-related quality of life in adult cancer survivors: A systematic review. Journal of Affective Disorders, 276, 159-168. https://doi.org/10.1016/j.jad. 2020.07.044

Mols, F., Vingerhoets, A. J. J. M., Coebergh, J. W. W., \& van de PollFranse, L. V. (2009). Well-being, posttraumatic growth and benefit finding in long-term breast cancer survivors. Psychology \& Health, 24(5), 583-595. https://doi.org/10.1080/0887044070 1671362

Morales, J. F., Moya, M., Gaviria, E., \& Cuadrado, I. (2007). Psicología Social [Social Psychology] (3rd ed.). McGraw Hill.

Neria, Y., Nandi, A., \& Galea, S. (2008). Post-traumatic stress disorder following disasters: A systematic review. Psychological Medicine, 38(4), 467-480. https://doi.org/10.1017/S0033291707001353

Páez, D., Reyes, C., \& Villagrán, L. (2012). Evaluación del Crecimiento Postraumático Colectivo en víctimas de catástrofes humanitarias. Universidad del País Vasco. 
Páez, D., Vázquez, C., Bosco, S., Gasparre, A., Iraurgi, I., \& Sezibera, V. (2011). Crecimiento post estrés y post trauma: Posibles aspectos positivos y beneficiosos de la respuesta a los hechos traumáticos. En D. Páez, C. Martín Beristain, J. L. González, N. Basabe, \& J. De Rivera (Eds.), Superando la violencia colectiva y construyendo cultura de paz (pp. 311-339). Fundamentos.

Páez, D., Vázquez, C., \& Echeburúa, E. (2013). Trauma social, afrontamiento comunitario y crecimiento postraumático colectivo [Social trauma, community coping and collective posttraumatic growth]. In M. J. Carrasco \& B. Charro (Eds.), Crisis, vulnerabilidad y superación [Crisis, vulnerability and growth]. Universidad de Comillas.

Powell, S., Rosner, R., Butollo, W., Tedeschi, R. G., \& Calhoun, L. G. (2003). Posttraumatic growth after war: A study with former refugees and displaced people in Sarajevo. Journal of Clinical Psychology, 59(1), 71-83. https://doi.org/10.1002/jclp.10117

Prieto-Ursúa, M., \& Jódar, R. (2020). Finding meaning in hell. The role of meaning, religiosity and spirituality in posttraumatic growth during the coronavirus crisis in Spain. Frontiers in psychology, 11, 567836. https://doi.org/10.3389/fpsyg.2020.567836

Rodríguez-Rey, R., \& Alonso-Tapia, J. (2019). Predicting posttraumatic growth in mothers and fathers of critically ill children: A longitudinal study. Journal of Clinical Psychology in Medical Settings, 26(3), 372-381. https://doi.org/10.1007/s10880-018-9594-3

Rodríguez-Rey, R., Garrido-Hernansaiz, H., \& Collado, S. (2020). Psychological impact and associated factors during the initial stage of the coronavirus (COVID-19) pandemic among the general population in Spain. Frontiers in Psychology, 11, 1540. https://doi.org/ 10.3389/fpsyg. 2020.01540

Salari, N., Hosseinian-Far, A., Jalali, R., Vaisi-Raygani, A., Rasoulpoor, S., Mohammadi, M., Rasoulpoor, S., \& Khaledi-Paveh, B. (2020). Prevalence of stress, anxiety, depression among the general population during the COVID-19 pandemic: A systematic review and meta-analysis. Globalization and Health, 16, 57. https://doi.org/10.1186/s12992-020-00589-w

Sawyer, A., Ayers, S., \& Field, A. P. (2010). Posttraumatic growth and adjustment among individuals with cancer or HIV/AIDS: A metaanalysis. Clinical Psychology Review, 30(4), 436-447. https://doi. org/10.1016/j.cpr.2010.02.004

Shakespeare-Finch, J., \& Lurie-Beck, J. (2014). A meta-analytic clarification of the relationship between posttraumatic growth and symptoms of posttraumatic distress disorder. Journal of Anxiety Disorders, 28(2), 223-229. https://doi.org/10.1016/j.janxdis.2013. 10.005

Taku, K., Calhoun, L. G., Tedeschi, R. G., Gil-Rivas, V., Kilmer, R. P., \& Cann, A. (2007). Examining posttraumatic growth among Japanese university students. Anxiety, Stress \& Coping, 20(4), 353-367. https://doi.org/10.1080/10615800701295007

Tedeschi, R. G., \& Calhoun, L. G. (1996). The Posttraumatic Growth Inventory: Measuring the positive legacy of trauma. Journal of
Traumatic Stress, 9(3), 455-471. https://doi.org/10.1002/jts.24900 90305

Tedeschi, R. G., Cann, A., Taku, K., Senol-Durak, E., \& Calhoun, L. G. (2017). The Posttraumatic Growth Inventory: A Revision Integrating Existential and Spiritual Change. Journal of Traumatic Stress, 30(1), 11-18. https://doi.org/10.1002/jts.22155

Vázquez, C., \& Páez, D. (2010). Posttraumatic growth in Spain. In T. Weiss \& R. Berger (Eds.), Posttraumatic growth and culturally competent practice: Lessons learned from around the globe (pp. 97-112). Wiley.

Vázquez, C., Valiente, C., García, F. E., Contreras, A., Peinado, V., Trucharte, A., \& Bentall, R. P. (2021). Post-Traumatic Growth and Stress-Related Responses During the COVID-19 Pandemic in a National Representative Sample: The Role of Positive Core Beliefs About the World and Others. Journal of happiness studies. Advance online publication. https://doi.org/10.1007/ s10902-020-00352-3

Vishnesky, T., Cann, A., Calhoun, L. G., Tedeschi, R. G., \& Demakis, G. J. (2010). Gender differences in self-reported posttraumatic growth: A meta-analysis. Psychology of Women Quarterly, 34(1), 110-120. https://doi.org/10.1111/j.1471-6402.2009.01546.x

Weiss D. S. (2007). The Impact of Event Scale: Revised. In J. P. Wilson \& C. S. Tang (Eds), International and cultural psychology: Cross-Cultural Assessment of Psychological Trauma and PTSD. Springer. https://doi.org/10.1007/978-0-387-70990-1_10

Weiss, T., \& Berger, R. (2006). Reliability and Validity of a Spanish Version of the Posttraumatic Growth Inventory. Research on Social Work Practice, 16(2), 191-199. https://doi.org/10.1177/ 1049731505281374

Wlodarczyk, A., Basabe, N., Páez, D., Reyes, C., Villagran, L., Madariaga, C., Palacio, J., \& Martínez, F. (2016). Communal coping and posttraumatic growth in a context of natural disasters in Spain, Chile, and Colombia. Cross-Cultural Research, 50(4), 325-355. https://doi.org/10.1177/1069397116663857

Wlodarczyk, A., Basabe, N., Páez, D., Villagrán, L., \& Reyes, C. (2017). Individual and collective posttraumatic growth in victims of natural disasters: A multidimensional perspective. Journal of Loss and Trauma, 22(5), 371-384. https://doi.org/10.1080/15325 024.2017.1297657

World Health Organization (2021, March). WHO Coronavirus (COVID-19) Dashboard. https://covid19.who.int/

Yu, Y., Yu, Y., \& Hu, J. (2021). COVID-19 among Chinese high school graduates: Psychological distress, growth, meaning in life and resilience. Journal of Health Psychology. Advance online publication. https://doi.org/10.1177/1359105321990819

Publisher's Note Springer Nature remains neutral with regard to jurisdictional claims in published maps and institutional affiliations. 